\title{
Conhecimento de enfermeiros de Neonatologia acerca do Cateter Venoso Central de Inserção Periférica
}

\author{
Neonatology nurses' knowledge about Peripherally Inserted Central Venous Catheter \\ Conocimiento de enfermeros de Neonatología sobre el Catéter Venoso Central de Inserción Periférica
}

\author{
Marcela Patricia Macêdo Belo', Roberta Albuquerque Mello de Castro Silva", \\ Isis Larissa Maia Nogueira", Daniele Pereira Mizoguti"', Claudiane Maria Urbano Ventura"I \\ 'Instituto de Medicina Integral Prof. Fernando Figueira, Recife-PE, Brasil. \\ "Faculdade Pernambucana de Saúde, Curso de Enfermagem. Recife-PE, Brasil. \\ III Instituto de Medicina Integral Prof. Fernando Figueira, Unidade de Terapia Intensiva Neonatal. Recife-PE, Brasil.
}

Submissão: 10-01-2011 Aprovação: 08-03-2012

\section{RESUMO}

O Cateter Central de Inserção Periférica (PICC) vem sendo utilizado como acesso venoso seguro para neonatos de risco. Objetivou-se descrever o conhecimento e prática dos enfermeiros das cinco unidades públicas de Terapia Intensiva Neonatal, de Recife-PE, sobre a utilização do PICC. A amostra foi composta por 52 enfermeiros; os dados foram coletados de janeiro a fevereiro de 2010. Nos resultados verificou-se que $64,8 \%$ dos enfermeiros não possuíam habilitação para inserção do PICC. Apenas duas unidades utilizavam o PICC rotineiramente. Sobre a indicação desse acesso, houve nível de acerto acima de $70 \%$. Na unidade B apenas 8,3\% dos enfermeiros referiram localização inicial adequada da ponta do cateter. Conclui-se que é necessário maior incentivo à capacitação dos enfermeiros para utilização do PICC.

Descritores: Cateterismo periférico; Recém-nascido; Terapia Intensiva Neonatal; Enfermagem Neonatal; Conhecimento.

ABSTRACT
The Peripherally Inserted Central Catheter (PICC) has been used as a safe venous access for infants at risk. The aim of this study was to describe the knowledge and practice of nurses from the five public Neonatal Intensive Care Units, of Recife-PE, Brazil, about the use of the PICC. The sample was comprised by 52 nurses; data were collected from January to February/2010. It was found that $64,8 \%$ of nurses did not have license for insertion of the PICC. Only two units routinely used the PICC. About the indication of the access, the accuracy was above $70 \%$. In unit B only $8,3 \%$ of nurses reported adequate initial location of the catheter tip. It was concluded that is necessary greater incentives to train nurses to use the PICC.

Key words: Catheterization, peripheral; Infant newborn; Intensive Care, Neonatal; Neonatal Nursing; Knowledge.

\section{RESUMEN}

El Catéter Central de Inserción Periférica (PICC) ha sido utilizado como un acceso venoso seguro para los niños en situación de riesgo. El objetivo de este estudio fue describir los conocimientos y la práctica de los enfermeros de cinco unidades públicas de Cuidados Intensivos Neonatales, en Recife-PE, Brasil, sobre el uso del PICC. La muestra comprendió 52 enfermeras; los datos fueron recolectados de enero a febrero de 2010 . Se encontró que $64,8 \%$ de las enfermeras no tenía licencia para la inserción del PICC. Sólo dos unidades estaban utilizando rutinariamente el PICC. En la indicación de acceso, nivel de precisión fue superior al 70\%. En la unidad B sólo el 8,3\% de las enfermeras reportó la ubicación inicial adecuado de la punta del catéter. Se concluye que es necesario fomentar una mayor potenciación de las enfermeras a usar el PICC.

Palabras clave: Cateterismo periférico; Recién nacido; Cuidado Intensivo Neonatal; Enfermería Neonatal; Conocimiento. 


\section{INTRODUÇÃO}

A Unidade de Terapia Intensiva constitui-se em ambiente terapêutico apropriado para o tratamento de pacientes cuja sobrevivência se encontra ameaçada por determinadas patologias ou situações que causam desequilíbrio e insuficiência de um ou mais sistemas orgânicos ${ }^{(1,2)}$.

A morbidade e a mortalidade no período neonatal atingem principalmente os recém-nascidos (RNs) pré-termos (idade gestacional inferior a 37 semanas) e/ou de baixo peso (peso ao nascimento inferior a $2.500 \mathrm{~g}$ ). Quando um bebê nasce prematuramente, seu desenvolvimento é interrompido, tornando-o vulnerável e, muitas vezes, levando-o a ser tratado em uma Unidade de Terapia Intensiva ${ }^{(2-5)}$.

A utilização de novas tecnologias tem contribuído para o aumento da sobrevida de neonatos com idade gestacional e peso de nascimento cada vez menores. Tal avanço exige uma equipe altamente especializada com conhecimento técnico-científico para prestar uma assistência qualificada a essa clientela diferenciada ${ }^{(6,7)}$.

Uma das áreas que tem exigido atenção na assistência de enfermagem em Neonatologia é a terapia intravenosa, pois a necessidade de administração de drogas vasoativas, hiperosmolares, antibióticos e nutrição parenteral exige a manutenção de um acesso venoso seguro e duradouro ${ }^{(7,8)}$.

O Cateter Central de Inserção Periférica (PICC) vem sendo utilizado como alternativa de acesso venoso estável e eficaz para neonatos criticamente enfermos. Trata-se de um cateter longo e flexível, inserido através de uma veia periférica que, por meio de uma agulha introdutora, progride até o terço distal da veia cava superior ou veia cava inferior, adquirindo dessa forma propriedades de acesso venoso centra|(9-11).

O primeiro relato da tentativa de acessar as veias centrais por intermédio de um cateter inserido perifericamente, embora de forma precária, foi descrito em $1929^{(9)}$. A eficácia do procedimento foi comprovada na década de 50, século $\mathrm{XX}$, quando se observou que as drogas que atingiam os grandes vasos, como a veia cava, tornavam-se mais diluídas, diminuindo os riscos de reações inflamatórias e trombose química, além de permitir um tratamento por tempo prolongado $^{(9-12)}$. Em Unidades de Terapia Intensiva Neonatal (UTIN) o PICC foi introduzido a partir de 1973 por Shaw, médico neonatologista ${ }^{(9,12)}$.

Nos Estados Unidos, na década de 80, houve o surgimento de programas de capacitação profissional de enfermeiros para a prática da instalação do PICC, que, associados aos avanços tecnológicos, tornou possível a expansão dessa técnica em ambientes hospitalares e, posteriormente, no cuidado domiciliar ${ }^{(11)}$. Na década de 90 estes cateteres passam a ser confeccionados com materiais mais biocompatíveis, menos trombogênicos, constituídos de poliuretano ou elastômeros de silicone, oferecendo maiores vantagens e benefícios aos pacientes e apresentando menores riscos de complicações ${ }^{(10)}$.

A partir de 1990, o Brasil passou a utilizar essa técnica e atualmente têm-se usado cada vez mais os PICCs, principalmente em $\mathrm{RNs}^{(8,12)}$. Esse procedimento tornou-se vantajoso, porque permite reduzir a frequência de punções intravenosas, consequentemente, minimiza procedimentos invasivos, o estresse e o desconforto do neonato ${ }^{(6-8)}$.

A competência técnica e legal para o Enfermeiro inserir o PICC encontra-se no artigo $1^{\circ}$ da Resolução no 258/2001, do Conselho Federal de Enfermagem (COFEN). De acordo com o artigo $2^{\circ}$ dessa resolução para o enfermeiro desempenhar tal atividade, deve estar qualificado e/ou capacitado profissionalmente ${ }^{(13)}$.

Considerando a importância do enfermeiro como sendo um dos principais responsáveis pela indicação, inserção, manutenção e retirada do cateter central de inserção periférica, este estudo tem como objetivo analisar o conhecimento e a prática desses profissionais nas cinco unidades públicas de Terapia Intensiva Neonatal, localizadas em Recife-PE.

\section{MÉTODOS}

Estudo descritivo, transversal, com abordagem quantitativa, com o objetivo de analisar o conhecimento e a prática dos enfermeiros acerca da utilização do PICC em recém-nascidos. O estudo foi realizado durante o período de janeiro e fevereiro de 2010, nas cinco unidades públicas de terapia intensiva neonatal, localizadas no município de Recife-PE: Centro de Saúde Amaury de Medeiros (CISAM), Hospital Agamenon Magalhães (HAM), Hospital Barão de Lucena (HBL), Hospital das Clínicas (HC) e o Instituto de Medicina Integral Prof. Fernando Figueira (IMIP). Os dados foram coletados através de um instrumento estruturado com questões fechadas elaboradas pelos próprios pesquisadores com base nas referências bibliográficas utilizadas. O questionário foi aplicado nos turnos e horários concordantes com a escala de trabalho de cada participante mediante presença de um dos pesquisadores. Foram abordadas questões sobre os conhecimentos gerais do PICC (indicações, contraindicações, vantagens, complicações, entre outras), sobre a técnica de inserção e manutenção do cateter, bem como as variáveis biológicas e profissionais que permitiram a caracterização da amostra.

A população foi composta por 60 enfermeiros que atuavam nas unidades de terapia intensiva neonatais, sendo incluídos no estudo 52 profissionais após a assinatura do Termo de Consentimento Livre e Esclarecido. Considerou-se como perda aqueles que estavam em licença médica, licença maternidade ou em período de férias, assim como os que se recusaram a participar do estudo.

A análise estatística foi realizada através do software Epi-Info 6.4. Foram utilizadas a distribuição de frequência simples e as medidas de tendência central, apresentadas em tabelas e gráficos.

Os aspectos éticos da pesquisa foram conduzidos de acordo com a resolução número 196/96 do Ministério da Saúde, que aprova as diretrizes e normas regulamentadas da pesquisa envolvendo seres humanos. O projeto de pesquisa foi aprovado pelo Comitê de Ética em Pesquisa em Seres Humanos do Instituto de Medicina Integral Professor Fernando Figueira, conforme Protocolo $\mathrm{n}^{\circ} 1.574$ de 18 de novembro/2009. 


\section{RESULTADOS}

Participaram do estudo 52 enfermeiros que atuam nas cinco unidades neonatais, em diferentes turnos, desses, $98,1 \%$ eram do sexo feminino. Obteve-se uma média de idade de 35,9 anos $( \pm 7,6)$. Foi evidenciado um perfil de enfermeiros com tempo médio de formação de 11,9 anos $( \pm 7,2)$ e de 7,2 anos $( \pm 5,6)$ de serviço dentro da UTIN. Dentre os sujeitos pesquisados, $85,2 \%$ têm curso de pós-graduação. Entretanto, $64,8 \%$ dos enfermeiros que atuam nas unidades de terapia intensiva neonatais não possuíam habilitação para inserção do PICC. Quando investigado em qual momento obtiveram conhecimento sobre esse tipo de acesso venoso, $88,9 \%$ responderam que foi atuando durante a vida profissional. Mediante questionamento às respectivas gerentes de cada Unidade Neonatal foi verificado que apenas as Unidades A e C utilizavam o PICC rotineiramente. Constatou-se também que, com exceção da unidade $B$, todas tinham protocolo
Gráfico 1. Frequência de enfermeiros que possuem habilitação para inserção do PICC e conhecimento acerca da existência do protocolo para utilização do PICC nas UTINs. Recife-PE, 2010.

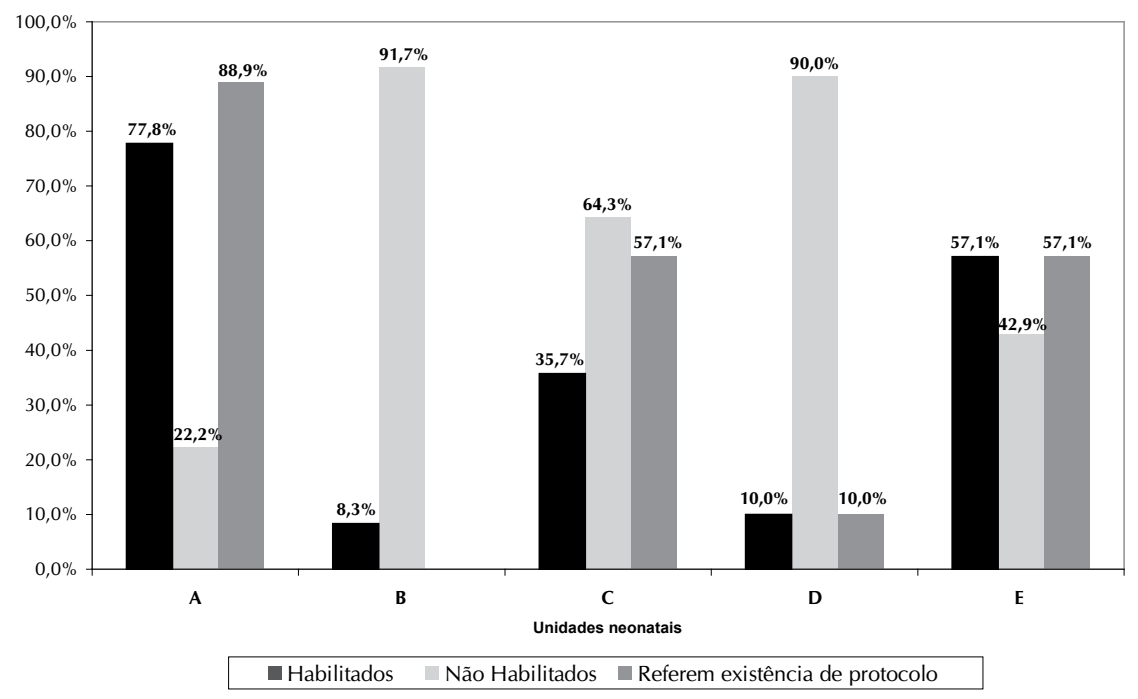

disponível para inserção do PICC. Porém, apenas 10\% dos profissionais da unidade $\mathrm{D}$ referiram a existência desse documento (Gráfico 1). Foi verificado também que apenas as Unidades A e C inseriam o PICC rotineiramente.

Tabela 1. Conhecimentos gerais dos enfermeiros das UTINs pesquisadas sobre o PICC. Recife-PE, 2010.

\begin{tabular}{|c|c|c|c|c|c|c|c|c|c|c|}
\hline \multirow{2}{*}{ Variáveis/Unidades neonatais } & \multicolumn{2}{|c|}{ A } & \multicolumn{2}{|c|}{ B } & \multicolumn{2}{|c|}{$\mathrm{C}$} & \multicolumn{2}{|c|}{ D } & \multicolumn{2}{|c|}{ E } \\
\hline & $\mathbf{n}$ & $\%$ & $\mathbf{n}$ & $\%$ & $\mathbf{n}$ & $\%$ & $\mathbf{n}$ & $\%$ & $\mathbf{n}$ & $\%$ \\
\hline \multicolumn{11}{|l|}{ Indicação: } \\
\hline -Acertos & 9 & 100 & 9 & 75,1 & 10 & 71,4 & 8 & 80,0 & 5 & 71,4 \\
\hline -Erros & - & - & 2 & 16,6 & 1 & 7,2 & 1 & 10,0 & 2 & 28,6 \\
\hline -Não sabe/Não respondeu & - & - & 1 & 8,3 & 3 & 21,4 & 1 & 10,0 & - & - \\
\hline \multicolumn{11}{|l|}{ Vantagens: } \\
\hline -Acertos & 6 & 66,7 & 8 & 66,7 & 8 & 57,2 & 2 & 20,0 & 4 & 57,1 \\
\hline -Erros & 3 & 33,3 & 4 & 33,3 & 3 & 21,4 & 7 & 70,0 & 2 & 28,6 \\
\hline -Não sabe/Não respondeu & - & - & - & - & 3 & 21,4 & 1 & 10,0 & 1 & 14,3 \\
\hline \multicolumn{11}{|l|}{ Contra-indicação: } \\
\hline -Acertos & 2 & 22,3 & 2 & 16,6 & 5 & 35,7 & 5 & 50,0 & 2 & 28,6 \\
\hline -Erros & 7 & 77,7 & 5 & 41,7 & 9 & 64,3 & 3 & 30,0 & 3 & 42,9 \\
\hline -Não sabe/Não respondeu & - & - & 5 & 41,7 & - & - & 2 & 20,0 & 2 & 28,5 \\
\hline \multicolumn{11}{|l|}{ Principais veias utilizadas: } \\
\hline -Acertos & 9 & 100 & 7 & 58,4 & 13 & 92,9 & 4 & 40,0 & 5 & 71,4 \\
\hline -Erros & - & - & 2 & 16,6 & - & - & 3 & 30,0 & 1 & 14,3 \\
\hline -Não sabe/Não respondeu & - & - & 3 & 25,0 & 1 & 7,1 & 3 & 30,0 & 1 & 14,3 \\
\hline \multicolumn{11}{|l|}{ Complicações: } \\
\hline -Acertos & 6 & 66,7 & 5 & 41,7 & 13 & 92,9 & 2 & 20,0 & 3 & 42,9 \\
\hline -Erros & 3 & 33,3 & 4 & 33,3 & - & - & 3 & 30,0 & 3 & 42,9 \\
\hline -Não sabe/Não respondeu & - & - & 3 & 25,0 & 1 & 7,1 & 5 & 50,0 & 1 & 14,3 \\
\hline \multicolumn{11}{|l|}{ Duração do PICC: } \\
\hline -Acertos & 9 & 100 & 6 & 50,1 & 11 & 78,6 & 4 & 40,0 & 4 & 57,1 \\
\hline -Erros & - & - & 4 & 33,3 & 1 & 7,1 & - & - & 1 & 14,3 \\
\hline -Não sabe/Não respondeu & - & - & 2 & 16,6 & 2 & 14,3 & 6 & 60,0 & 2 & 28,6 \\
\hline Total & 9 & 100 & 12 & 100 & 14 & 100 & 10 & 100 & 7 & 100 \\
\hline
\end{tabular}


Tabela 2. Conhecimento dos enfermeiros das UTINs pesquisadas sobre a técnica do PICC. Recife-PE, 2010.

\begin{tabular}{|c|c|c|c|c|c|c|c|c|c|c|}
\hline \multirow{2}{*}{ Variáveis/Unidades neonatais } & \multicolumn{2}{|c|}{ A } & \multicolumn{2}{|c|}{ B } & \multicolumn{2}{|c|}{$\mathrm{C}$} & \multicolumn{2}{|c|}{ D } & \multicolumn{2}{|c|}{$\mathbf{E}$} \\
\hline & $\mathbf{n}$ & $\%$ & $\mathrm{n}$ & $\%$ & $\mathbf{n}$ & $\%$ & $\mathbf{n}$ & $\%$ & $\mathbf{n}$ & $\%$ \\
\hline \multicolumn{11}{|l|}{ Monitorização do RN: } \\
\hline -Acertos & 8 & 88,9 & 9 & 75,1 & 14 & 100 & 6 & 60,0 & 9 & 100 \\
\hline -Erros & 1 & 11,1 & 1 & 8,3 & - & - & - & - & - & - \\
\hline -Não sabe/Não respondeu & - & - & 2 & 16,6 & - & - & 4 & 40,0 & - & - \\
\hline \multicolumn{11}{|l|}{ Posição do RN para inserção do PICC: } \\
\hline -Acertos & 4 & 44,4 & 1 & 8,3 & 4 & 28,6 & - & - & 2 & 28,6 \\
\hline -Erros & 5 & 55,6 & 7 & 58,4 & 9 & 64,3 & 2 & 20,0 & 5 & 71,4 \\
\hline -Não sabe/Não respondeu & - & - & 4 & 33,3 & 1 & 7,1 & 8 & 80,0 & - & - \\
\hline \multicolumn{11}{|l|}{ Mensuração do Cateter: } \\
\hline -Acertos & 5 & 55,6 & 2 & 16,6 & 6 & 42,8 & 1 & 10,0 & 2 & 28,6 \\
\hline -Erros & 4 & 44,4 & 2 & 16,6 & 4 & 28,6 & - & - & 3 & 42,8 \\
\hline -Não sabe/Não respondeu & - & - & 8 & 66,8 & 4 & 28,6 & 9 & 90,0 & 2 & 28,6 \\
\hline \multicolumn{11}{|l|}{ Localização inicial da ponta do cateter: } \\
\hline -Acertos & 7 & 77,8 & 1 & 8,3 & 7 & 50,0 & 1 & 10,0 & 2 & 28,6 \\
\hline -Erros & 2 & 22,2 & 4 & 33,3 & 2 & 14,3 & 1 & 10,0 & 4 & 57,1 \\
\hline -Não sabe/Não respondeu & - & - & 7 & 58,4 & 5 & 35,7 & 8 & 80,0 & 1 & 14,3 \\
\hline Total & 9 & 100 & 12 & 100 & 14 & 100 & 10 & 100 & 7 & 100 \\
\hline
\end{tabular}

Tabela 3. Conhecimento dos enfermeiros das UTINs pesquisadas sobre cuidados com o PICC. Recife-PE, 2010.

\begin{tabular}{|c|c|c|c|c|c|c|c|c|c|c|}
\hline \multirow{2}{*}{ Variáveis/Unidades neonatais } & \multicolumn{2}{|c|}{$\mathbf{A}$} & \multicolumn{2}{|c|}{ B } & \multicolumn{2}{|c|}{ C } & \multicolumn{2}{|c|}{ D } & \multicolumn{2}{|c|}{$\mathbf{E}$} \\
\hline & $\mathbf{n}$ & $\%$ & $\mathrm{n}$ & $\%$ & $\mathbf{n}$ & $\%$ & $\mathbf{n}$ & $\%$ & $\mathbf{n}$ & $\%$ \\
\hline \multicolumn{11}{|l|}{ Tipo de seringa utilizada: } \\
\hline -Acertos & 9 & 100 & 1 & 8,3 & 13 & 92,9 & 2 & 20,0 & 5 & 71,4 \\
\hline -Erros & - & - & 8 & 66,7 & - & - & - & - & - & - \\
\hline -Não sabe/Não respondeu & - & - & 3 & 25,0 & 1 & 7,1 & 8 & 80,0 & 2 & 28,6 \\
\hline \multicolumn{11}{|l|}{ Troca $1^{\circ}$ curativo: } \\
\hline -Acertos & 7 & 77,8 & 9 & 75,1 & 11 & 78,6 & 3 & 30,0 & 5 & 71,4 \\
\hline -Erros & 1 & 11,1 & 2 & 16,6 & 1 & 7,1 & 3 & 30,0 & - & - \\
\hline -Não sabe/Não respondeu & 1 & 11,1 & 1 & 8,3 & 2 & 14,3 & 4 & 40,0 & 2 & 28,6 \\
\hline \multicolumn{11}{|l|}{ Troca dos demais curativos: } \\
\hline -Acertos & 9 & 100 & 7 & 58,4 & 12 & 85,7 & 6 & 60,0 & 5 & 71,4 \\
\hline -Erros & - & - & 4 & 33,3 & 1 & 7,1 & 1 & 10,0 & - & 28,6 \\
\hline -Não sabe/Não respondeu & - & - & 1 & 8,3 & 1 & 7,1 & 3 & 30,0 & 2 & - \\
\hline Total & 9 & 100 & 12 & 100 & 14 & 100 & 10 & 100 & 7 & 100 \\
\hline
\end{tabular}

Na Tabela 1 estão apresentados os resultados que abordam os conhecimentos gerais sobre o PICC. Em relação à indicação, houve um nível de acerto acima de $70 \%$, em todas as instituições. Já no item que abrange as contraindicações para utilização desse cateter, apenas na instituição $\mathrm{D}$, metade dos profissionais respondeu corretamente, nas demais unidades foram constatados índices abaixo de $36 \%$. Sobre as principais veias de escolha para inserção do PICC e o tempo de permanência do acesso para terapêutica intravenosa, todas as Unidades
Neonatais obtiveram um índice de acerto igual ou superior a $50 \%$, exceto a Unidade D, onde $40 \%$ dos pesquisados souberam responder ambas as questões. Referente às vantagens e às complicações, $80 \%$ dos participantes da Unidade D não sabiam ou não responderam a alternativa correta. Por outro lado, na instituição C 92,9\% dos enfermeiros souberam identificar as principais complicações da utilização do PICC em neonatos.

Sobre a técnica relacionada ao cateter (tabela 2), observou-se que, quanto à monitorização do recém-nascido, durante 
a inserção do PICC, os níveis de acertos foram iguais ou superiores a $60 \%$, destacando as Unidades C e E que atingiram $100 \%$ de acerto. Entretanto, a posição do recém-nascido para proceder à introdução do cateter, foi citada corretamente por $8,3 \%$ dos enfermeiros da instituição B. Ressalta-se que a unidade $\mathrm{D}$ não obteve nenhum acerto nessa questão, pois $20 \%$ erraram e $80 \%$ não souberam responder. Referente à técnica de mensuração e posição inicial da ponta do cateter, a instituição A obteve índice de acerto de 55,6\% e 77,8\% respectivamente. Salienta-se que, na Unidade B, apenas $16,6 \%$ dos enfermeiros sabiam como medir corretamente o cateter para proceder à inserção e só $8,3 \%$ dos pesquisados na mesma Unidade sabiam identificar a correta posição inicial da ponta do cateter.

Em relação aos cuidados com o PICC (tabela 3), em geral o presente estudo identificou índices acima de $70 \%$ na maioria dos itens pesquisados. No entanto, apenas 8,3\% dos sujeitos na Unidade B e $20 \%$ na Unidade D acertaram quanto à seringa utilizada para infusões nestes tipos de cateteres. A respeito da troca do primeiro curativo, na Unidade D 30\% dos enfermeiros acertaram o período correto. No item relacionado as demais trocas de curativos, destaca-se a Unidade A e C com $100 \%$ e $85,7 \%$ de acertos respectivamente.

\section{DISCUSSÃO}

A aplicação de cuidados neonatais requer alta qualidade, com técnicas, habilidades e conhecimentos novos e a equipe de enfermagem deve estar ciente das tecnologias existentes e disponíveis no mercado ${ }^{(14)}$.

A terapêutica intravenosa é essencial para a manutenção da vida no período neonatal. Contudo, um dos fatores dificultadores na prestação dessa assistência em RNs é a limitação de sua rede venosa, tornando o acesso venoso periférico um dos procedimentos mais difíceis de ser realizado. Dessa forma, requer do executante conhecimento de anatomia e técnica com o objetivo de promover o sucesso do procedimento e prolongar a permanência do acesso venoso ${ }^{(7)}$.

Mesmo com o conhecimento tardio de informações sobre o PICC durante a formação profissional, a Unidade A apresentou percentual elevado de enfermeiros habilitados, esse índice reflete a busca dessa categoria profissional pelo aprofundamento dos seus conhecimentos teóricos e práticos. Por outro lado, na instituição $C$, onde também, existe inserção desse tipo de cateter, apenas $35,7 \%$ tinham habilitação para essa técnica. Nas demais Unidades, foi constatado que não existia a prática regular de inserção do PICC, apesar de, na Unidade $\mathrm{E}$, mais da metade dos profissionais serem habilitados.

Um fator importante observado foi o desconhecimento por parte de alguns enfermeiros das Unidades Neonatais sobre a existência de protocolo para inserção do PICC nas instituições, sabe-se que, exceto a unidade $B$, todas tinham protocolo disponível.

A existência e utilização de protocolos que direcionam a prática de enfermagem no emprego desse cateter visa padronizar condutas e melhorar a qualidade na assistência, o que se torna fundamental para o êxito da prática com o $\mathrm{PICC}^{(11,15)}$.
Em relação à indicação desse tipo de cateter, a maioria dos participantes respondeu de acordo com a literatura, ou seja, a necessidade de obter e manter um acesso venoso profundo por tempo prolongado, administração de soluções hiperosmolares, vesicantes e irritantes ${ }^{(16)}$.

De maneira contraditória, no item que aborda as contraindicações para utilização do PICC, constatou-se que houve índice de acerto igual ou inferior a 50\% na maioria das Unidades participantes. Considera-se que as contraindicações estão relacionadas com a administração de grandes volumes em "bolus" e sob pressão, presença de lesões cutâneas no local de inserção, infecção de pele ou tecido subcutâneo no local ou próximo ao local proposto para inserção, alterações anatômicas estruturais e venopunção ou dissecção prévia ${ }^{(17)}$.

No presente estudo, percebeu-se que a maioria dos enfermeiros de quatro instituições referiram corretamente as principais veias utilizadas, bem como a duração recomendada para utilização da terapêutica intravenosa com o PICC, o que não ocorreu na instituição D.

As veias dos membros superiores são consideradas os principais vasos de escolha para inserção do cateter em recém-nascidos, pela facilidade de acesso, por possuírem menor número de válvulas e por oferecerem menor risco de complicações $^{(6,9)}$. Com relação ao tempo de permanência desse tipo de acesso, o recomendado pela literatura é que seja mantido até o final da terapia intravenosa, exceto em situações em que ocorram o aparecimento de sinais flogísticos no sítio de inserção ou ao longo do trajeto venoso, trombose ou obstrução ${ }^{(6,9)}$.

Quando questionado sobre as vantagens desse cateter $80 \%$ dos enfermeiros da Unidade D não responderam ou não souberam a resposta correta, nas outras Unidades existiu um nível de acerto acima de $57 \%$. As principais vantagens da inserção do cateter PICC são a confiabilidade de acesso seguro, menor risco de infecção em relação a outros dispositivos vasculares centrais, mantém preservados os demais acessos venosos, diminuição do estresse causado pelas múltiplas punções, inserção menos traumática, menor risco de ocorrer flebite química, extravasamento de fluidos, maior tempo de permanência e redução dos $\operatorname{custos}^{(11,16)}$.

A verificação das complicações relacionadas à infecção é de grande responsabilidade do enfermeiro, pois a monitorização dos sinais como hipertermia, rubor e secreção no óstio da inserção devem ser realizados diariamente. Evidenciando a importância de profissionais treinados e familiarizados com a técnica do PICC para que possam intervir nas complicações precocemente $^{(18)}$. As principais complicações relacionadas à pós-inserção do cateter são: flebite, celulite, infecção sistêmica, oclusão e migração do cateter, fratura desse com potencial risco de embolia e trombose ${ }^{(17)}$. Foi verificado que $66,7 \%$ dos enfermeiros da Unidade A e 92,9 da Unidade C demonstraram conhecer as principais complicações do PICC.

O processo de inserção deve contar com técnica de barreira máxima, dentre os cuidados fundamentais prévios na implantação do PICC em neonato estão: a monitorização cardiorrespiratória, a sedação e analgesia para prevenção da dor. A posição do RN deve ser decúbito dorsal, mantendo preferencialmente, o membro superior direito em ângulo de 
$90^{\circ}$ em relação ao tórax, mensurar com fita métrica o local de inserção do cateter até a altura da clavícula, e desse ponto até o $3^{\circ}$ espaço intercostal direito onde a ponta do cateter deve ter sua visualização radiográfica no terço distal da veia cava superior ${ }^{(12)}$.

Ao pesquisar o conhecimento a cerca da técnica de implantação do PICC foi verificado que quanto à monitorização cardiorrespiratória do $\mathrm{RN}$ durante o procedimento, de forma geral, as respostas foram condizentes com a literatura. Já em relação ao posicionamento do neonato para proceder à introdução do cateter foi identificado que menos da metade dos profissionais responderam adequadamente em todas as instituições pesquisadas. Destaca-se que nenhum enfermeiro da Unidade D respondeu corretamente esse item.

A respeito da técnica de mensuração do PICC e a localização inicial da ponta do cateter ocorreu percentual de acerto maior pelos profissionais da instituição A, seguidos pelos da C. Aparentemente, o melhor desempenho dos enfermeiros dessas instituições pode estar relacionado à utilização do PICC na rotina dessas Unidades, o que não se observa nas demais Unidades, já que a prática era esporádica ou inexistente.

Quanto à troca do primeiro curativo do PICC, com exceção da Unidade D, as Unidades Neonatais apresentaram acerto acima de $70 \%$. No que concerne à troca dos curativos subsequentes, destaca-se a Unidade $\mathrm{A}$, em que todos os enfermeiros mostraram conhecimento quanto à manutenção de um meio apropriado para a prevenção de complicações locais e/ ou sistêmicas relacionadas ao cateter.

Sabe-se que o curativo possui duas funções: a criação de um ambiente que proteja o local de inserção do cateter e a de evitar o seu deslocamento. Portanto, recomenda-se que a primeira troca do curativo seja realizada após 24 horas da colocação do cateter e as subsequentes a cada 7 dias ou antes se ficar úmido, solto ou apresentar qualquer outra condição que comprometa a sua condição estéril ${ }^{(18)}$.
Na administração de medicação em "bolus" recomendam-se seringas de $10 \mathrm{cc}$ e de $20 \mathrm{cc}$, pois estas exercem menor pressão, evitando o rompimento e embolia no cateter $^{(17-19)}$. Observou-se que um profissional da Unidade B e dois da Unidade $\mathrm{D}$ demonstraram conhecimento sobre a seringa utilizada para infusões no PICC.

\section{CONSIDERAÇÕES FINAIS}

Conclui-se, portanto, que o cateter central de inserção periférica é um avanço tecnológico nas Unidades de Terapia Intensiva Neonatal, proporcionando diversas vantagens ao recém nascido, principalmente aqueles de alto risco. Entretanto, apesar dos benefícios dessa técnica, grande parcela dos enfermeiros pesquisados não tinha habilitação para inserção do PICC e apenas duas Unidades Neonatais utilizavam esse cateter na prática diária.

A partir dos resultados desse estudo, observou-se que o conhecimento geral sobre o PICC apresentado pelos enfermeiros das Unidades pesquisadas foi predominantemente satisfatório. Contudo, os profissionais que tinham habilitação para esse tipo de cateter central e o inseriam rotineiramente obtiveram melhores resultados em relação ao conhecimento da técnica de inserção, manutenção e retirada desse acesso venoso. Evidenciando dessa forma a importância do conhecimento teórico aliado a prática.

Dessa forma, faz-se necessário o maior incentivo à capacitação dos enfermeiros para utilização do PICC, através de instituições idôneas, a fim de garantir a melhoria constante da qualidade da assistência aos neonatos. Este estudo teve como possível fator limitador o fato de o questionário ter sido composto apenas por questões fechadas podendo não ter inferido a total profundidade dos dados, salienta-se a necessidade de realização de mais estudos com metodologias diversas sobre a temática exposta, para que sejam aprimorados os conhecimentos sobre os procedimentos inerentes a utilização do PICC.

\section{REFERÊNCIAS}

1. Kamada I, Rocha SMM, Barbeira CBS. Internações em unidades de terapia intensiva neonatal no Brasil 1998 2001. Rev Latinoam Enferm 2003;11(4):436-43.

2. Pailaquilén RMB, Maldonado YM, Toro YU, Mora CC, Manríquez GS. Tendência da mortalidade infantil e dos neonatos menores de 32 semanas e de muito baixo peso. Rev Latinoam Enferm 2011;19(4):977-84.

3. Almeida MFB, Guinsburg R, Martinez FE, Prociano RS, Leone CR, Marba STM, Rugolo LMSS, Luz JH, Lopes. Perinatal factors associated with early deaths of preterm infants born in Brazilian Network on Neonatal Research centers . J Pediatr 2008;84(4):300-7.

4. Bezerra AM, Tavares MM, Santos ACF. Uso do Cateter Central por Inserção Periférica em Neonatologia. In: Rodrigues FPM, Magalhães M. Normas e Condutas em Neonatologia. São Paulo: Atheneu; 2008. p. 337-45.
5. Ceccon MEJR. O peso do recém-nascido como fator de risco para morbidade e mortalidade: como interpretar?. Pediatria 2007;29(3):162-64.

6. Freitas EM, Nunes ZB. O enfermeiro na práxis de cateter central de inserção periférica em neonato. REME Rev Min Enferm 2009;13(2):215-24.

7. Lourenço SA, Kakehashi TY. Avaliação da implantação do cateter venoso central de inserção periférica em neonatologia. Acta Paul Enferm 2003;16(2):26-32.

8. Rodrigues ZS, Chaves EMC, Cardoso MVLML. Atuação do enfermeiro no cuidado com o cateter central de inserção periférica no recém-nascido. Rev Bras Enferm 2006;59(5):626-9.

9. Silva GRG, Nogueira MFH. Terapia intravenosa em recém-nascidos: orientações para o cuidado de enfermagem. Rio de Janeiro: Cultura Médica; 2004. p.23-37. 
10. Camargo PP. Procedimento de inserção, manutenção e remoção do cateter central de inserção periférica em neonatos. São Paulo. Dissertação. [Mestrado em Enfermagem Obstétrica e Neonatal] - São Paulo: Escola de Enfermagem da Universidade de São Paulo; 2007.

11. Vendramim P, Pedreira MLG, Peterlini MAS. Cateteres centrais de inserção periférica em crianças de hospitais do município de São Paulo. Rev Gaúch Enferm 2007;28(3):331-39.

12. Alencar LFA. Acesso Venoso Central em recém-nascidos: inserção periférica versus dissecção venosa. Recife. Dissertação. [Mestrado em Saúde da Criança e do Adolescente] - Universidade Federal de Pernambuco; 2005.

13. Conselho Federal de Enfermagem [homepage na internet]. Resolução no 258 de 12 de julho de 2001. Inserção de Cateter Periférico Central, pelos Enfermeiros. [acesso em 20 jun 2009]. Disponível em: http://www.portalcofen. gov.br/legislação/r258.htm.

14. Lima FD. A escolha do dispositivo de cateterização venosa periférica: contribuições para o cuidado de enfermagem. Rio de Janeiro. Dissertação [Mestrado em Enfermagem] - Escola de Enfermagem Alfredo Pinto. Universidade
Federal do Estado do Rio de Janeiro; 2009.

15. Sakita NK. Cateterismo central por inserção periférica em UTI neonatal de nível terciário: incidência de complicações e fatores de risco associados. São Paulo. Dissertação. [Mestrado em Ciências - Pediatria] - Faculdade de Medicina da Universidade de São Paulo; 2009.

16. Secretaria de Saúde do Rio de Janeiro [homepage na internet]. Rotina para cateter venoso central de inserção periférica em neonatos. Rio de Janeiro; 2002. [acesso em 20 jun 2009]. Disponível em: http://www.saude.rj.gov.br/ Docs/cecih/Picc.doc.

17. Jesus VC, Secoli SR. Complicações acerca do cateter venoso central de inserção periférica (PICC). Ciênc Cuid Saúde 2007;6(2):252-60.

18. Câmara SMC, Tavares TJL, Chaves EMC. Cateter Venoso Central de Inserção Periférica: análise do uso em recém-nascidos de uma unidade neonatal publica em Fortaleza. Rev RENE 2007;8(1):26-31.

19. Franceschi AT, Cunha MLC. Eventos adversos relacionados ao uso de cateteres venosos centrais em recém-nascidos hospitalizados. Rev Latinoam Enferm 2010;18(2):57-63. 